TRANSACTIONS OF THE

AMERICAN MATHEMATICAL SOCIETY

Volume 355, Number 10, Pages 4125-4136

S 0002-9947(03)03339-7

Article electronically published on June 18, 2003

\title{
THE STRUCTURE OF EQUICONTINUOUS MAPS
}

\author{
JIE-HUA MAI
}

\begin{abstract}
Let $(X, d)$ be a metric space, and $f: X \rightarrow X$ be a continuous map. In this paper we prove that if $R(f)$ is compact, and $\omega(x, f) \neq \emptyset$ for all $x \in X$, then $f$ is equicontinuous if and only if there exist a pointwise recurrent isometric homeomorphism $h$ and a non-expanding map $g$ that is pointwise convergent to a fixed point $v_{0}$ such that $f$ is uniformly conjugate to a subsystem $(h \times g) \mid S$ of the product map $h \times g$. In addition, we give some still simpler necessary and sufficient conditions of equicontinuous graph maps.
\end{abstract}

\section{INTRODUCTION}

Let $(X, d)$ be a metric space. A map $f: X \rightarrow X$ is said to be equicontinuous (with respect to $d$ ) if there exists a function $\eta:(0, \infty) \rightarrow(0, \infty)$ satisfying the following condition:

(E) For any $\varepsilon>0$, any $n \in \mathbf{N}(\equiv\{1,2, \cdots\})$ and any $x, y \in X$ with $d(x, y) \leq$ $\eta(\varepsilon)$, one has $\eta(\varepsilon) \leq \varepsilon$ and $d\left(f^{n}(x), f^{n}(y)\right) \leq \varepsilon$.

It is interesting to find some characterizations of equicontinuous maps. In [1], by means of the orbit map $O_{f}: X \rightarrow C(\mathbf{N}, X)$ and the metric $d_{f}$, Akin, Auslander and Berg gave some necessary and sufficient conditions for a continuous map $f$ of a compact metric space $(X, d)$ to be equicontinuous. Recently, Blanchard, Host and Maass [2] discussed the topological complexity, and showed that a continuous surjection $f$ of a compact metric space $X$ is equicontinuous if and only if any finite open cover of $X$ under $f$ has bounded complexity.

On one-dimensional spaces, one has some still finer results. In [7], Cano proved that if $f$ is an equicontinuous map from the interval $I=[0,1]$ to itself, then the set $\operatorname{Fix}(f)$ of fixed points of $f$ is connected, and furthermore, if $\operatorname{Fix}(f)$ is nondegenerate, then $f$ has no periodic points except fixed points. Bruckner and $\mathrm{Hu}$ (only if) and Boyce (if) proved that a continuous map $f: I \rightarrow I$ is equicontinuous if and only if $\bigcap_{n=0}^{\infty} f^{n}(I)=\operatorname{Fix}\left(f^{2}\right)$; see [6] and [5]. This result was also proved by Blokh in [4]. Valaristos [10] described the characters of equicontinuous circle maps: A continuous map $f$ of the unit circle $S^{1}$ to itself is equicontinuous if and only if one of the following four statements holds: (1) $f$ is topologically conjugate to a rotation; (2) $\operatorname{Fix}(f)$ contains exactly two points and $\operatorname{Fix}\left(f^{2}\right)=S^{1} ;(3) \operatorname{Fix}(f)$ contains exactly

Received by the editors March 4, 2002 and, in revised form, November 1, 2002.

2000 Mathematics Subject Classification. Primary 54E40, 54H20; Secondary 37B20, 37E25.

Key words and phrases. Metric space, equicontinuous map, recurrent point, uniform conjugacy, graph map.

Project supported by the Special Foundation of National Prior Basic Researches of China (Grant No. G1999075108). 
one point and $\operatorname{Fix}\left(f^{2}\right)=\bigcap_{n=1}^{\infty} f^{n}\left(S^{1}\right)$; (4) $\operatorname{Fix}(f)=\bigcap_{n=1}^{\infty} f^{n}\left(S^{1}\right)$. Recently, Sun [9] obtained some necessary and sufficient conditions of equicontinuous $\sigma$-maps.

In this paper we will study equicontinuous maps of general metric spaces. Our aim is to find some new conditions of equivalence and to describe the structure of equicontinuous maps. Our main results are the following theorems:

Theorem 3.2. Let $(X, d)$ be a metric space, and $f: X \rightarrow X$ be a continuous map. Suppose $R(f)$ is compact, and $\omega(x, f) \neq \emptyset$ for all $x \in X$. Then $f$ is equicontinuous if and only if there exists a unique retraction $\gamma: X \rightarrow R(f)$ such that $\gamma f=f \gamma$, and $\lim _{n \rightarrow \infty} d\left(f^{n}(x), f^{n} \gamma(x)\right)=0$ for all $x \in X$, and there exists a metric $d^{\prime}$ on $X$ uniformly equivalent to $d$ such that, under $d^{\prime}, f$ and $\gamma$ are non-expanding, and $f \mid R(f)$ is isometric.

Theorem 4.1. Let $(X, d)$ be a metric space, and $f: X \rightarrow X$ be a continuous map. Suppose that $R(f)$ is compact, and $\omega(x, f) \neq \emptyset$ for all $x \in X$. Then $f$ is equicontinuous if and only if there exist a pointwise recurrent isometric homeomorphism $h$ and $a$ non-expanding map $g$ that is pointwise convergent to a fixed point $v_{0}$ such that $f$ is uniformly conjugate to a subsystem $(h \times g) \mid S$ of the product map $h \times g$.

Theorem 5.2. Let $G$ be a connected graph with a metric d, and let $f: G \rightarrow G$ be a continuous map. Then the following four statements are equivalent:

(i) $f$ is equicontinuous;

(ii) $\bigcap_{n=1}^{\infty} f^{n}(G)=R(f)$;

(iii) $f \mid \bigcap_{n=1}^{\infty} f^{n}(G)$ is equicontinuous;

(iv) $f \mid \cap_{n=1}^{\infty} f^{n}(G)$ is a periodic homeomorphism, or is topologically conjugate to an irrational rotation of the unit circle $S^{1}$.

\section{Properties of equicontinuous maps}

Let $(X, d)$ be a metric space, and $f: X \rightarrow X$ be a continuous map. For $x \in V \subset X$ and $r \geq 0$, write $B(x, r)=B(x, r, d)=\{y \in X: d(y, x) \leq r\}$, $B(V, r)=B(V, r, d)=\{y \in X: d(y, V) \leq r\}, O(x, f)=\left\{f^{n}(x): n=0,1,2, \cdots\right\}$, and $\omega(x, f)=\bigcap_{n=0}^{\infty} \overline{O\left(f^{n}(x), f\right)} . O(x, f)$ and $\omega(x, f)$ are called the orbit and the $\omega$-limit set of $x$ under $f$, respectively. $x$ is a recurrent point of $f$ if $x \in \omega(x, f)$. $x$ is a non-wandering point of $f$ if for any $\varepsilon>0$ there exists $m=m(\varepsilon) \in \mathbf{N}$ such that $f^{m}(B(x, \varepsilon)) \cap B(x, \varepsilon) \neq \emptyset$. $x$ is an almost periodic point of $f$ if for any $\varepsilon>0$ there exists $m=m(\varepsilon) \in \mathbf{N}$ such that $d\left(x,\left\{f^{n+i}(x): i=0,1, \cdots, m\right\}\right)<\varepsilon$ for all $n \in \mathbf{N}$. Denote by $\Omega(f)$ (resp. $R(f)$, resp. $A P(f)$ ) the set of all non-wandering (resp. recurrent, resp. almost periodic) points of $f . f$ is said to be pointwise recurrent if $R(f)=X$. $f$ is said to be periodic if there exists $k \in \mathbf{N}$ such that $f^{k}$ is the identity map on $X$. Note that $\Omega(f)$ is a closed subset of $X$.

Proposition 2.1. Let $f: X \rightarrow X$ be an equicontinuous map. Then $R(f)=\Omega(f)$, and hence $R(f)$ is a closed subset of $X$.

Proof. It suffices to show that $R(f)=\Omega(f)$. If $R(f) \neq \Omega(f)$, then there exist $x \in \Omega(f)-R(f)$ and $\varepsilon>0$ such that $d(x, O(f(x), f))>2 \varepsilon$. Let $\eta:(0, \infty) \rightarrow(0, \infty)$ be a function that satisfies the condition (E). Write $\delta=\eta(\varepsilon)$. Then there exist $y \in B(x, \delta)$ and $n \in \mathbf{N}$ such that $f^{n}(y) \in B(x, \delta)$, which implies $d\left(f^{n}(x), f^{n}(y)\right)>$ $2 \varepsilon-\delta \geq \varepsilon$. But this contradicts the condition (E). Thus we must have $R(f)=$ $\Omega(f)$. 
Let $V$ be a subset of $X$. $V$ is called an $f$-invariant set or an invariant set of $f$ if $f(V) \subset V$. $V$ is called a minimal set of $f$ if it is nonempty, closed and $f$-invariant and it does not contain any proper subset having these three properties. Note that all points in a minimal set are recurrent.

Proposition 2.2. Let $f: X \rightarrow X$ be an equicontinuous map, and $x \in X$. Suppose $\omega(x, f) \neq \emptyset$. Then $\omega(x, f)$ is a minimal set.

Proof. Note that $\omega(x, f)$ is closed and $f$-invariant. If $\omega(x, f)$ is not minimal, then there exist $v$ and $w \in \omega(x, f)$ such that $w \notin \overline{O(v, f)}$. Let $\varepsilon=d(w, O(v, f)) / 2$, and let $\eta:(0, \infty) \rightarrow(0, \infty)$ be as above. Take $y \in O(x, f)$ such that $d(y, v)<\eta(\varepsilon)$. Then, for any $n \in \mathbf{N}$, we have $d\left(f^{n}(y), O(v, f)\right) \leq \varepsilon$, which implies $d\left(f^{n}(y), w\right) \geq \varepsilon$, and hence $w \notin \omega(y, f)=\omega(x, f)$. This is a contradiction. Thus $\omega(x, f)$ must be a minimal set.

Note that if $x \in R(f)$, then $\omega(x, f)=\overline{O(x, f)} \neq \emptyset$. From Proposition 2.2 we get

Corollary 2.1. Let $f: X \rightarrow X$ be an equicontinuous map. Then $\overline{O(x, f)}$ is a minimal set if and only if $x \in R(f)$.

It is well known (for example, see [3]) that if $X$ is compact, then $\overline{O(x, f)}$ is a minimal set if and only if $x \in A P(f)$. Thus from Corollary 2.1 we obtain

Corollary 2.2. Let $f: X \rightarrow X$ be an equicontinuous map. If $X$ is compact, then $R(f)=A P(f)$.

Let $(X, d)$ and $(Y, \rho)$ be metric spaces. A map $g: X \rightarrow Y$ is said to be nonexpanding (resp. isometric) if $\rho(g(x), g(y)) \leq d(x, y)$ (resp. $\rho(g(x), g(y))=$ $d(x, y))$ for all $x, y \in X$. On non-expanding maps and isometric maps, we have the following proposition, with a proof suggested by the referee.

Proposition 2.3. Let $f: X \rightarrow X$ be an equicontinuous map, If $R(f)$ is compact, and $f \mid R(f)$ is non-expanding, then $f \mid R(f): R(f) \rightarrow R(f)$ is an isometric homeomorphism.

Proof. We may suppose $X=R(f)$. Then $f$ is an injection. (In fact, if $f(v)=f(w)$ for some $v, w \in X$, then $v$ and $w$ are in the same minimal set. Equicontinuity then implies that the point $(v, w) \in X \times X$ is recurrent for the product map $f \times f$, which clearly implies $v=w$.) So $f$ is a homeomorphism, and it is well known (and easily proved) that all $(x, y) \in X \times X$ are recurrent (in fact almost periodic) under $f \times f$. From this the isometry property follows immediately.

Proposition 2.4. Let $f: X \rightarrow X$ be an equicontinuous map. If $X$ is compact, then $\bigcap_{n=1}^{\infty} f^{n}(X)=R(f)$.

Proof. Since $X$ is compact, we have $f(R(f))=R(f)$, which implies $\bigcap_{n=1}^{\infty} f^{n}(X) \supset$ $R(f)$. Conversely, for any $x_{0} \in \bigcap_{n=1}^{\infty} f^{n}(X)$, there exist points $y_{1}, y_{2}, \cdots$ in $X$ such that $f^{n}\left(y_{n}\right)=x_{0}$ for all $n \in \mathbf{N}$. Let $\eta:(0, \infty) \rightarrow(0, \infty)$ be as above. Since $X$ is compact, there exist integers $0<k_{1}<k_{2}<\cdots$ and $y_{0} \in X$ such that $d\left(y_{k_{n}}, y_{0}\right) \leq$ $\eta(1 / n)$, for all $n \in \mathbf{N}$, which implies $d\left(f^{k_{n}}\left(y_{0}\right), x_{0}\right)=d\left(f^{k_{n}}\left(y_{0}\right), f^{k_{n}}\left(y_{k_{n}}\right)\right) \leq 1 / n$. Thus, by Proposition 2.2, we have $x_{0} \in \omega\left(y_{0}, f\right) \subset R(f)$. Hence $\bigcap_{n=1}^{\infty} f^{n}(X)=$ $R(f)$.

Note that every continuous map from a compact metric space to itself is uniformly continuous. The following proposition is obvious. 
Proposition 2.5. Suppose $X$ is compact, and $f: X \rightarrow X$ is continuous. Then

(1) For any given $k \in \mathbf{N}, f^{k}$ is equicontinuous if and only if $f$ is equicontinuous.

(2) If $X_{0} \subset X$ is an $f$-invariant set, and there exists $m \in \mathbf{N}$ such that $f^{m}(X) \subset X_{0}$, then $f$ is equicontinuous if and only if $f \mid X_{0}$ is equicontinuous.

\section{Structure of Equicontinuous maps}

Let $(X, d)$ and $(Y, \rho)$ be metric spaces. A bijection $h: X \rightarrow Y$ is called a uniform homeomorphism if both $h$ and $h^{-1}$ are uniformly continuous.

Let $f: X \rightarrow X$ and $g: Y \rightarrow Y$ be continuous maps. $f$ and $g$ are said to be uniformly conjugate if there exists a uniform homeomorphism $h: X \rightarrow Y$ such that $h f=g h$. Such an $h$ is called a uniform conjugacy from $f$ to $g$. Note that if $(X, d)$ is compact, then every homeomorphism from $X$ to $Y$ (if it exists) is a uniform homeomorphism, and every topological conjugacy from $f$ to $g$ is a uniform conjugacy.

Let $d$ and $d^{\prime}$ be two metrics on $X$. $d^{\prime}$ and $d$ are said to be uniformly equivalent if the identity map $i d_{X}:(X, d) \rightarrow\left(X, d^{\prime}\right)$ is a uniform homeomorphism.

Evidently, we have

Lemma 3.1. Let $(X, d)$ and $(Y, \rho)$ be metric spaces, and $f: X \rightarrow X$ and $g$ : $Y \rightarrow Y$ be uniformly conjugate maps. Then $f$ is equicontinuous if and only if $g$ is equicontinuous. In particular, suppose $d$ and $d^{\prime}$ are two uniformly equivalent metrics on a set $X$. Then a map $f: X \rightarrow X$ is equicontinuous with respect to $d$ if and only if it is equicontinuous with respect to $d^{\prime}$.

The following lemma is due to Akin, Auslander and Berg [1].

Lemma 3.2. Let $(X, d)$ be a metric space, and $f:(X, d) \rightarrow(X, d)$ be an equicontinuous map. Define another metric $d_{f}$ on $X$ by

$$
d_{f}(x, y)=\sup \left\{d\left(f^{n}(x), f^{n}(y)\right): n=0,1,2, \cdots\right\}, \quad \text { for all } x, y \in X .
$$

Then $d_{f}$ is uniformly equivalent to $d$.

Let $W \subset X$. A continuous map $\gamma: X \rightarrow W$ is called a retraction if $\gamma(w)=w$ for each $w \in W$.

Theorem 3.1. Let $(X, d)$ be a metric space, and $f:(X, d) \rightarrow(X, d)$ be an equicontinuous map. Suppose that $R(f)$ is compact. Then

(1) Under the metric $d_{f}, f$ is non-expanding, and $f \mid R(f): R(f) \rightarrow R(f)$ is an isometric homeomorphism.

(2) If $\omega(x, f) \neq \emptyset$ for all $x \in X$, then there exists a unique retraction $\gamma: X \rightarrow$ $R(f)$ such that $\gamma f=f \gamma$,

$$
\lim _{n \rightarrow \infty} d\left(f^{n}(x), f^{n}(\gamma(x))\right)=0, \quad \text { for all } x \in X,
$$

and, under $d_{f}$, $\gamma$ is non-expanding.

Proof. (1) It follows from (3.1) that, under the metric $d_{f}, f$ is non-expanding, and hence, by Proposition 2.3, $f \mid R(f): R(f) \rightarrow R(f)$ is an isometric homeomorphism.

(2) For any given $x \in X$, since $\omega(x, f) \neq \emptyset$, it follows from Proposition 2.2 and Lemma 3.2 that there exist $y \in \omega(x, f) \subset R(f)$ and integers $0<n_{1}<n_{2}<\cdots$ such that $d_{f}\left(f^{n_{i}}(x), y\right)<2^{-i}$ for all $i \in \mathbf{N}$. By (1) of this theorem, for any $i \in \mathbf{N}$, there is a unique point $w_{i} \in R(f)$ such that $f^{n_{i}}\left(w_{i}\right)=y$. Since $R(f)$ is compact, the sequence $w_{1}, w_{2}, \cdots$ has a subsequence converging to a point $v=v_{x} \in R(f)$. 
For convenience, we may assume that the sequence $w_{1}, w_{2}, \cdots$ itself converges to $v$ and that $d_{f}\left(w_{i}, v\right)<2^{-i}$ for all $i \in \mathbf{N}$. Therefore, for any $j \geq n_{i}$, we have

$$
\begin{aligned}
d_{f}\left(f^{j}(x), f^{j}(v)\right) \leq d_{f}\left(f^{n_{i}}(x), f^{n_{i}}(v)\right) & \leq d_{f}\left(f^{n_{i}}(x), y\right)+d_{f}\left(f^{n_{i}}\left(w_{i}\right), f^{n_{i}}(v)\right) \\
& \leq 2^{-i}+d_{f}\left(w_{i}, v\right) \leq 2^{1-i},
\end{aligned}
$$

which with Lemma 3.2 implies $\lim _{n \rightarrow \infty} d\left(f^{n}(x), f^{n}(v)\right)=\lim _{n \rightarrow \infty} d_{f}\left(f^{n}(x), f^{n}(v)\right)$ $=0$. Put $\gamma(x)=v=v_{x}$. Then we obtain a map $\gamma: X \rightarrow R(f)$ that satisfies (3.2). For any $u \in R(f)-\left\{v_{x}\right\}$, it follows that $\lim _{n \rightarrow \infty} d_{f}\left(f^{n}(x), f^{n}(u)\right)=$ $\lim _{n \rightarrow \infty} d_{f}\left(f^{n}(v), f^{n}(u)\right)=d_{f}(v, u)>0$, which implies $\liminf _{n \rightarrow \infty} d\left(f^{n}(x), f^{n}(u)\right)$ $>0$. Thus the map $\gamma: X \rightarrow R(f)$ satisfying (3.2) is unique. From (3.2) we obtain

$$
\lim _{n \rightarrow \infty} d\left(f^{n}(f(x)), f^{n}(f \gamma(x))\right)=0, \quad \text { for all } x \in X,
$$

which with the uniqueness of $\gamma$ yields $\gamma f=f \gamma$. For any $x, y \in X$, it is easy to see that

$$
\begin{aligned}
d_{f}(\gamma(x), \gamma(y)) & =\lim _{n \rightarrow \infty} d_{f}\left(f^{n} \gamma(x), f^{n} \gamma(y)\right) \\
& =\lim _{n \rightarrow \infty} d_{f}\left(f^{n}(x), f^{n}(y)\right) \leq d_{f}(x, y) .
\end{aligned}
$$

Hence $\gamma$ is continuous, and under $d_{f}, \gamma$ is non-expanding. Obviously, we have $\gamma(w)=w$ for all $w \in R(f)$. Thus $\gamma$ is a retraction. Theorem 3.1 is proven.

Remark 3.1. If $(X, d)$ is compact, and $f: X \rightarrow X$ is equicontinuous, then, by Proposition 2.1 , the conditions " $R(f)$ is compact" and " $\omega(x, f) \neq \emptyset$ for all $x \in X$ " in Theorem 3.1 automatically hold.

A topological property $\mathrm{P}$ is said to be retract-preserving if each retract of a topological space with property $\mathrm{P}$ also has property $\mathrm{P}$. For example, it is easy to check that the compactness, the connectedness, the path connectedness, the local connectedness, the local path conectedness, the simple connectedness, the property that the $n$-th homotopy group is trivial, the contractibility, and the fixed point property are all retract-preserving properties. By Theorem 3.1, we have

Corollary 3.1. Let $(X, d)$ be a metric space, and $f: X \rightarrow X$ be an equicontinuous map. Suppose $R(f)$ is compact, and $\omega(x, f) \neq \emptyset$ for all $x \in X$. If $X$ has some retract-preserving property, then $R(f)$ also has this property.

From Corollary 3.1 we see that, for example, if $D$ is a disk in the Euclidean plane $\mathbf{R}^{2}$, and $f: D \rightarrow D$ is an equicontinuous map, then $R(f)$ is locally path connected and contractible.

Combining Theorem 3.1 with Lemmas 3.1 and 3.2 we obtain the following theorem, which gives a description of the structure of equicontinuous maps.

Theorem 3.2. Let $(X, d)$ be a metric space, and $f: X \rightarrow X$ be a continuous map. Suppose $R(f)$ is compact, and $\omega(x, f) \neq \emptyset$ for all $x \in X$. Then $f$ is equicontinuous if and only if there exists a unique retraction $\gamma: X \rightarrow R(f)$ such that $\gamma f=f \gamma$, and $\lim _{n \rightarrow \infty} d\left(f^{n}(x), f^{n} \gamma(x)\right)=0$ for all $x \in X$, and there exists a metric $d^{\prime}$ on $X$ uniformly equivalent to $d$ such that, under $d^{\prime}, f$ and $\gamma$ are non-expanding, and $f \mid R(f)$ is isometric.

It is well known (for example, see [1]) that each transitive equicontinuous map from a compact metric space $(X, d)$ onto itself is uniformly conjugate to an isometric homeomorphism. This property is also true for non-transitive maps. In fact, from Theorem 3.2 and Propositions 2.4 and 2.3 we can obtain the following corollary. 
Corollary 3.2. A map from a compact metric space onto itself is equicontinuous if and only if it is uniformly conjugate to an isometric homeomorphism.

\section{Products of Equicontinuous maps}

Let $(X, d)$ and $(Y, \rho)$ be metric spaces, and $W \subset X$ and $V \subset Y$ be nonempty sets. Define a metric $D_{d \rho}=\max \{d, \rho\}$ on the cartesian product $W \times Y$ by (4.1)

$$
D_{d \rho}((w, v),(x, y))=\max \{d(w, x), \rho(v, y)\}, \quad \text { for all }(w, v) \text { and }(x, y) \in W \times Y \text {. }
$$

Let $h: W \rightarrow W$ and $g: V \rightarrow V$ be continuous maps. $g$ is said to be pointwise convergent to a point $v_{0} \in V$ if $\lim _{n \rightarrow \infty} g^{n}(y)=v_{0}$ for every $y \in V$. Define $h \times g:\left(W \times V, D_{d \rho}\right) \rightarrow\left(W \times V, D_{d \rho}\right)$, which is called the product of $h$ and $g$, by

$$
(h \times g)(w, v)=(h(w), g(v)) \text {, for all }(w, v) \in W \times V .
$$

Let $S$ be a nonempty subset of $W \times V$. If $S$ is an invariant set of $h \times g$, then the restriction $(h \times g) \mid S: S \rightarrow S$ is called a subsystem of $h \times g$. Obviously, we have

Lemma 4.1. If $h: W \rightarrow W$ is a pointwise recurrent isometric homeomorphism, and $g: V \rightarrow V$ is a non-expanding map pointwise convergent to a point $v_{0} \in V$, then every subsystem of $h \times g$ is equicontinuous.

Let $\beta \geq 1$ be a constant. A continuous map $f: X \rightarrow X$ is said to be $\beta$ equicontinuous with respect to the metric $d$ if $d\left(f^{n}(x), f^{n}(y)\right) \leq \beta d(x, y)$ for all $x, y \in X$ and all $n \in \mathbf{N}$. Note that $f$ is 1-equicontinuous if and only if $f$ is non-expanding. A retraction $\gamma: X \rightarrow W$ is called a vertical projection relative to $d$ if it is non-expanding, and

$$
d(x, w)=\max \{d(x, \gamma(x)), d(\gamma(x), w)\}, \quad \text { for any } x \in X \text { and any } w \in W .
$$

Let $d^{\prime}$ be another metric on $X$. $d^{\prime}$ and $d$ are said to be $\beta$-equivalent if

$$
d(x, y) / \beta \leq d^{\prime}(x, y) \leq \beta d(x, y), \quad \text { for all } x, y \in X .
$$

Lemma 4.2. Let $(X, d)$ be a metric space, $W$ be a nonempty subset of $X$, and $\beta \geq 1$ be a constant. Suppose that $\gamma: X \rightarrow W$ is a non-expanding retraction, $f: X \rightarrow X$ is a $\beta$-equicontinuous map with respect to $d, f(W)=W$, and $f \mid W$ is isometric. Then there exists a metric $d^{\prime}$ on $X$ that has the following properties:

(i) $d^{\prime}$ and $d$ are 2-equivalent;

(ii) $\gamma$ is a vertical projection relative to $d^{\prime}$;

(iii) $f$ is $4 \beta$-equicontinuous with respect to $d^{\prime}$;

(iv) Under $d^{\prime}, f \mid W$ is still isometric.

Proof. Denote by $\mathbf{R}^{X}$ the set of all maps from $X$ to the real axis $\mathbf{R}$. Define a metric $\rho$ on $\mathbf{R}^{X}$ by

$$
\rho(\varphi, \psi)=\sup \{|\varphi(u)-\psi(u)|: u \in X\}, \quad \text { for all } \varphi, \psi \in \mathbf{R}^{X} .
$$

Note that we permit $\rho(\varphi, \psi)=\infty$ for some $\varphi, \psi \in \mathbf{R}^{X}$. But in the following only the case $\rho(\varphi, \psi)<\infty$ is considered. Let the metric $D_{d \rho}=\max \{d, \rho\}$ on the cartesian product $W \times \mathbf{R}^{X}$ be defined as above. Let $\lambda:(X, d) \rightarrow\left(\mathbf{R}^{X}, \rho\right)$ be defined by $\lambda(x)=\varphi_{x}$, for any $x \in X$, where $\varphi_{x} \in \mathbf{R}^{X}$ is defined by

$$
\varphi_{x}(y)=d(y, x), \quad \text { for all } y \in X .
$$


It is easy to see that $\lambda$ is an isometric imbedding. For any $\varphi, \psi \in \mathbf{R}^{X}$, let $\varphi-\psi \in \mathbf{R}^{X}$ be defined by $(\varphi-\psi)(y)=\varphi(y)-\psi(y)$, for all $y \in X$. Define maps $\xi=\lambda-\lambda \gamma$ : $X \rightarrow \mathbf{R}^{X}$ and $\mu=(\gamma, \xi): X \rightarrow W \times \mathbf{R}^{X}$ by, for all $x \in X$,

$$
\begin{gathered}
\xi(x)=\lambda(x)-\lambda \gamma(x)=\varphi_{x}-\varphi_{\gamma(x)}, \\
\mu(x)=(\gamma(x), \xi(x)) .
\end{gathered}
$$

Then, for any $x, y \in X$, we have

$$
D_{d \rho}(\mu(x), \mu(y))=\max \{d(\gamma(x), \gamma(y)), \rho(\lambda(x)-\lambda \gamma(x), \lambda(y)-\lambda \gamma(y))\} .
$$

Note that $\gamma \mid W=i d_{W}$, and $\gamma^{2}=\gamma$. It follows from (4.8) that

$$
\begin{gathered}
D_{d \rho}(\mu(x), \mu(y)) \leq \max \{d(x, y), \rho(\lambda(x), \lambda(y))+\rho(\lambda \gamma(x), \lambda \gamma(y))\} \\
=\max \{d(x, y), d(x, y)+d(\gamma(x), \gamma(y))\} \leq 2 d(x, y), \\
D_{d \rho}(\mu(x), \mu(y)) \geq \max \{d(\gamma(x), \gamma(y)), \rho(\lambda(x), \lambda(y))-\rho(\lambda \gamma(x), \lambda \gamma(y))\} \\
=\max \{d(\gamma(x), \gamma(y)), d(x, y)-d(\gamma(x), \gamma(y))\} \geq d(x, y) / 2, \\
D_{d \rho}(\mu(x), \mu(y)) \geq d(\gamma(x), \gamma(y))=D_{d \rho}(\mu \gamma(x), \mu \gamma(y)), \\
D_{d \rho}(\mu(x), \mu(y))=\max \{d(\gamma(x), y), d(x, \gamma(x))\}, \quad \text { if } \quad y \in W,
\end{gathered}
$$

and

$$
D_{d \rho}(\mu(x), \mu(y))=d(x, y), \quad \text { if }\{x, y\} \subset W \text { or } \gamma(x)=\gamma(y) .
$$

Define a metric $d^{\prime}$ on $X$ by

$$
d^{\prime}(x, y)=D_{d \rho}(\mu(x), \mu(y)), \quad \text { for all } x, y \in X .
$$

Then, by (4.9) and (4.10), $d^{\prime}$ and $d$ are 2-equivalent metrics. This implies that

$$
d^{\prime}\left(f^{n}(x), f^{n}(y)\right) / 2 \leq d\left(f^{n}(x), f^{n}(y)\right) \leq \beta d(x, y) \leq 2 \beta d^{\prime}(x, y)
$$

for all $x, y \in X$ and $n \in \mathbf{N}$. Thus $f$ is $4 \beta$-equicontinuous with respect to $d^{\prime}$. It follows from (4.14) and (4.13) that, under $d^{\prime}, f \mid W$ is still isometric. By (4.14) and (4.11), under $d^{\prime}, \gamma$ is still non-expanding. By (4.14), (4.12) and (4.13), we have

$$
d^{\prime}(x, w)=\max \left\{d^{\prime}(\gamma(x), w), d^{\prime}(x, \gamma(x))\right\}, \quad \text { for any } x \in X \text { and any } w \in W .
$$

Therefore, $\gamma$ is a vertical projection relative to $d^{\prime}$. Lemma 4.2 is proven.

We now give the main theorem in this section.

Theorem 4.1. Let $(X, d)$ be a metric space, and $f: X \rightarrow X$ be a continuous map. Suppose that $R(f)$ is compact, and $\omega(x, f) \neq \emptyset$ for all $x \in X$. Then $f$ is equicontinuous if and only if there exist a pointwise recurrent isometric homeomorphism $h$ and $a$ non-expanding map $g$ that is pointwise convergent to a fixed point $v_{0}$ such that $f$ is uniformly conjugate to a subsystem $(h \times g) \mid S$ of the product map $h \times g$.

Proof. The sufficiency is clear, by Lemma 4.1. We now show the necessity. Suppose that $f$ is equicontinuous. Let $d_{f}$ be the same as defined by (3.1). By Lemma 3.2, $d$ is uniformly equivalent to $d_{f}$. Let $W=R(f)$, and let the retraction $\gamma: X \rightarrow W$ be as in Theorem 3.1. Then $\gamma f=f \gamma, \lim _{n \rightarrow \infty} d\left(f^{n}(x), f^{n} \gamma(x)\right)=0$ for all $x \in X$, and under $d_{f}, \quad f$ and $\gamma$ are non-expanding, and $f \mid W: W \rightarrow W$ is an isometric homeomorphism. Therefore, there exists a metric $d^{\prime}$ on $X$ that is 2-equivalent to $d_{f}$ and has the properties (ii)-(iv) in Lemma 4.2. For the convenience of statement, 
we may replace $d^{\prime}$ by $d$, that is, we may assume that the following properties hold:

(ii) $^{\prime} \gamma$ is a vertical projection relative to $d$;

(iii) $^{\prime} f$ is 4-equicontinuous with respect to $d$;

(iv) ${ }^{\prime}$ under $d, f \mid W$ is isometric.

Let the metric $\rho$ on $\mathbf{R}^{X}$ and maps $\lambda:(X, d) \rightarrow\left(\mathbf{R}^{X}, \rho\right)$ and $\xi=\lambda-\lambda \gamma:$ $(X, d) \rightarrow\left(\mathbf{R}^{X}, \rho\right)$ be the same as in the proof of Lemma 4.2. For any nonnegative integer $n$ and any given $x, y \in X$ with $x \neq y, d(x, \gamma(x)) \leq d(y, \gamma(y))$ and $y \notin W$, write $x_{n}=f^{n}(x), y_{n}=f^{n}(y)$, and

$$
\begin{gathered}
a_{n}=d\left(x_{n}, \gamma\left(x_{n}\right)\right), \quad b_{n}=d\left(y_{n}, \gamma\left(y_{n}\right)\right), \quad s_{n}=d\left(x_{n}, y_{n}\right), \quad t_{n}=d\left(\gamma\left(x_{n}\right), \gamma\left(y_{n}\right)\right), \\
p_{n}=d\left(x_{n}, \gamma\left(y_{n}\right)\right), \quad q_{n}=d\left(y_{n}, \gamma\left(x_{n}\right)\right) .
\end{gathered}
$$

Then $s_{n} \geq t_{n}, s_{0}>0, b_{0}>0$, and $b_{0} \geq a_{0}$. It follows from (4.3) that $p_{n}=$ $\max \left\{a_{n}, t_{n}\right\}$, and $q_{n}=\max \left\{b_{n}, t_{n}\right\}$. Noting that $\lambda$ is an isometric imbedding, by (4.6), (4.4), (4.5) and properties (ii)' and (iii)' we get

$$
\begin{aligned}
& \rho(\xi(x), \xi(y))=\rho\left(\varphi_{x}-\varphi_{\gamma(x)}, \varphi_{y}-\varphi_{\gamma(y)}\right) \geq\left(\varphi_{x}-\varphi_{\gamma(x)}-\varphi_{y}+\varphi_{\gamma(y)}\right)(y) \\
&=s_{0}+b_{0}-q_{0} \geq \min \left\{s_{0}, b_{0}\right\}>0 \\
& \rho\left(\xi\left(x_{n}\right), \xi\left(y_{n}\right)\right)= \rho\left(\varphi_{x_{n}}-\varphi_{\gamma\left(x_{n}\right)}, \varphi_{y_{n}}-\varphi_{\gamma\left(y_{n}\right)}\right) \\
& \leq \min \left\{\rho\left(\varphi_{x_{n}}, \varphi_{y_{n}}\right)+\rho\left(\varphi_{\gamma\left(x_{n}\right)}, \varphi_{\gamma\left(y_{n}\right)}\right)\right. \\
&\left.\quad \rho\left(\varphi_{x_{n}}, \varphi_{\gamma\left(x_{n}\right)}\right)+\rho\left(\varphi_{y_{n}}, \varphi_{\gamma\left(y_{n}\right)}\right)\right\} \\
&= \min \left\{s_{n}+t_{n}, a_{n}+b_{n}\right\} \leq 4 \min \left\{s_{0}+t_{0}, a_{0}+b_{0}\right\} \\
& \leq 8 \min \left\{s_{0}, b_{0}\right\}
\end{aligned}
$$

and by (3.2) and $\gamma^{2}=\gamma$ we obtain

$$
\lim _{n \rightarrow \infty} \rho\left(\xi\left(y_{n}\right), \xi \gamma\left(y_{n}\right)\right)=\lim _{n \rightarrow \infty} \rho\left(\lambda\left(y_{n}\right), \lambda \gamma\left(y_{n}\right)\right)=0 .
$$

Denote by $v_{0}$ the origin of $\mathbf{R}^{X}$, whose definition is $v_{0}(x)=0$ for all $x \in X$. Then $\xi(x)=v_{0}$ if and only if $x \in W$. Write $V=\xi(X)$. It follows from (4.15) that $\xi \mid(X-W)$ is an injection. Thus we can define a map $g: V \rightarrow V$ by $g\left(v_{0}\right)=v_{0}$ and $g(v)=\xi f \xi^{-1}(v)$ for all $v \in V-\left\{v_{0}\right\}$. It is easy to see from (4.15) and (4.16) that $g$ is 8-equicontinuous with respect to $\rho$, and from (4.17) that $g$ is pointwise convergent to $v_{0}$. Let the metric $D_{d \rho}=\max \{d, \rho\}$ on $W \times \mathbf{R}^{X}$ and the map $\mu=(\gamma, \xi): X \rightarrow W \times V\left(\subset W \times \mathbf{R}^{X}\right)$ be the same as in the proof of Lemma 4.2. Let $S=\mu(X)$, and let $h=f \mid W:(W, d) \rightarrow(W, d)$. By property (iv)' with $W=R(f), h$ is a pointwise recurrent isometric homeomorphism. Noting $\gamma f=h \gamma$ and $\xi f=g \xi$, which imply $\mu f=(\gamma f, \xi f)=(h \gamma, g \xi)=(h \times g) \mu$, from (4.9) and (4.10) we see that $\mu$ is a uniform conjugacy from $f:(X, d) \rightarrow(X, d)$ to the subsystem $(h \times g) \mid S:\left(S, D_{d \rho}\right) \rightarrow\left(S, D_{d \rho}\right)$ of the product map $h \times g:$ $\left(W \times V, D_{d \rho}\right) \rightarrow\left(W \times V, D_{d \rho}\right)$. Define a metric $\rho_{g}$ on $V$ by

$$
\rho_{g}(u, v)=\sup \left\{\rho\left(g^{n}(u), g^{n}(v)\right): n=0,1,2, \cdots\right\}, \quad \text { for all } u, v \in V .
$$

Then, by Lemma 3.2 and Theorem 3.1, $\rho_{g}$ is uniformly equivalent to $\rho$, and $g:\left(V, \rho_{g}\right) \rightarrow\left(V, \rho_{g}\right)$ is non-expanding. Put $D_{d \rho_{g}}=\max \left\{d, \rho_{g}\right\}$. Then $f:(X, d) \rightarrow$ $(X, d)$ is still uniformly conjugate to $(h \times g) \mid S:\left(S, D_{d \rho_{g}}\right) \rightarrow\left(S, D_{d \rho_{g}}\right)$. Theorem 4.1 is proven. 


\section{EQUiCONTINUOUS GRAPH MAPS}

For several special spaces $X$, we have some still simpler conditions for a map $f: X \rightarrow X$ to be equicontinuous.

Theorem 5.1. Let $(X, d)$ be a metric space, and $f: X \rightarrow X$ be a uniformly continuous map. If there exists a nonempty invariant set $X_{0}$ of $f$ such that $f \mid X_{0}$ is equicontinuous, and there exist a finite set $V \subset X_{0}$ and positive integers $n_{1}<$ $n_{2}<\cdots$ such that

$$
f^{n_{k}}(X) \subset X_{0} \cup B\left(V, 2^{-k}\right) \quad \text { for any } k \in \mathbf{N},
$$

then $f$ is equicontinuous.

Proof. Let

$$
c=\min \{d(x, w):\{x, w\} \subset V \cup f(V) \text {, and } x \neq w\} .
$$

Then $c>0$. For any given $\varepsilon \in(0, c / 3]$, since $f$ is uniformly continuous, and $f \mid X_{0}: X_{0} \rightarrow X_{0}$ is equicontinuous, there exists $\varepsilon^{\prime} \in(0, \varepsilon]$ such that

$$
f\left(B\left(x, \varepsilon^{\prime}\right)\right) \subset B(f(x), \varepsilon), \quad \text { for all } x \in X,
$$

and

$$
f^{i}\left(B\left(x, \varepsilon^{\prime}\right) \cap X_{0}\right) \subset B\left(f^{i}(x), \varepsilon\right), \quad \text { for all } x \in X_{0} \text { and all } i \in \mathbf{N} .
$$

Take $k \in \mathbf{N}$ such that $2^{-k}<\varepsilon^{\prime} / 2$, and

$$
f\left(B\left(x, 2^{1-k}\right)\right) \subset B\left(f(x), \varepsilon^{\prime}\right), \quad \text { for all } x \in X .
$$

Since each of $f^{2}, f^{3}, \cdots$ is also uniformly continuous, there exists $\delta \in\left(0,2^{-k}\right]$ such that

$$
f^{i}(B(x, \delta)) \subset B\left(f^{i}(x), 2^{-k}\right), \quad \text { for all } x \in X \text { and all } i \in\left\{1, \cdots, n_{k}\right\} .
$$

Consider any given points $y, z \in X$ with $d(y, z) \leq \delta$. Write $y_{i}=f^{i}(y)$ and $z_{i}=f^{i}(z)$, for all $i \in \mathbf{N}$. It follows from (5.6) that

$$
d\left(y_{i}, z_{i}\right) \leq 2^{-k}, \quad \text { for } i=1, \cdots, n_{k} .
$$

Case 1. If $\left\{y_{n_{k}}, z_{n_{k}}\right\} \subset X_{0}$, then by (5.7) and (5.4) we have

$$
d\left(y_{i}, z_{i}\right) \leq \varepsilon, \quad \text { for all } i \geq n_{k} .
$$

Case 2. If $\left\{y_{n_{k}}, z_{n_{k}}\right\} \not \subset X_{0}$, then, noting $2^{1-k}<\varepsilon^{\prime} \leq \varepsilon \leq c / 3$, from (5.1), (5.7) and (5.2) we see that there exists a unique point $v \in V$ such that $\left\{y_{n_{k}}, z_{n_{k}}\right\}-X_{0} \subset B\left(v, 2^{-k}\right)$, which with (5.7) implies that

$$
\left\{y_{n_{k}}, z_{n_{k}}\right\} \subset B\left(v, 2^{1-k}\right), \quad \text { for some } v \in V .
$$

For $i \geq n_{k}$, write $v_{i}=f^{i-n_{k}}(v)$. Note that

$$
f^{i}(X) \subset f^{n_{k}}(X) \subset X_{0} \cup B\left(V, 2^{-k}\right), \quad \text { for all } i \geq n_{k} .
$$

Subcase 2.1. If $y_{n_{k}} \in X_{0}$, then, similar to (5.8), we have

$$
d\left(y_{i}, v_{i}\right) \leq \varepsilon, \quad \text { for all } i \geq n_{k} .
$$

Subcase 2.2. We now assume $y_{n_{k}} \notin X_{0}$.

Claim. If there exists an integer $\lambda \geq n_{k}$ such that $\left\{y_{j}: j=n_{k}, \cdots, \lambda\right\} \cap X_{0}=\emptyset$ and $\left\{v_{j}: j=n_{k}, \cdots, \lambda\right\} \subset V$, then

$$
d\left(y_{j}, v_{j}\right) \leq 2^{-k} \quad \text { for } j=n_{k}, \cdots, \lambda .
$$

Furthermore, if $v_{\lambda+1} \notin V$, then $y_{\lambda+1} \in X_{0}$. 
Proof of claim. (5.12) is clear, by (5.9), (5.10) and (5.5). Furthermore, if $v_{\lambda+1} \notin V$, then by (5.12), (5.5) and (5.2) we get $d\left(y_{\lambda+1}, v_{\lambda+1}\right) \leq \varepsilon^{\prime}<c-\varepsilon<d\left(v_{\lambda+1}, V\right)-2^{-k}$, which implies $d\left(y_{\lambda+1}, V\right)>2^{-k}$, and hence, by (5.10), we have $y_{\lambda+1} \in X_{0}$.

From the claim we see that

(a) If there exists an integer $\lambda \geq n_{k}$ such that $\left\{y_{j}: j=n_{k}, \cdots, \lambda\right\} \cap X_{0}=\emptyset$ and $y_{\lambda+1} \in X_{0}$, then $\left\{v_{j}: j=n_{k}, \cdots, \lambda\right\} \subset V$, and from (5.12), (5.5) and (5.4) we get

$$
\begin{cases}d\left(y_{j}, v_{j}\right) \leq 2^{-k}, & \text { for } j=n_{k}, \cdots, \lambda \\ d\left(y_{\lambda+1}, v_{\lambda+1}\right) \leq \varepsilon^{\prime} ; & \text { for all } i \geq \lambda+2 .\end{cases}
$$

(b) If $y_{j} \notin X_{0}$ for all integers $j \geq n_{k}$, then $v_{j} \in V$ for all $j \geq n_{k}$, and

$$
d\left(y_{j}, v_{j}\right) \leq 2^{-k}, \quad \text { for all } j \geq n_{k} .
$$

Combining (5.11), (5.13) and (5.14) we get

$$
d\left(y_{i}, v_{i}\right) \leq \varepsilon, \quad \text { for all } i \geq n_{k},
$$

no matter whether $y_{n_{k}} \in X_{0}$ or not. Similarly, we have

$$
d\left(z_{i}, v_{i}\right) \leq \varepsilon, \quad \text { for all } i \geq n_{k} .
$$

Combining (5.7), (5.8), (5.15) and (5.16), we obtain

$$
d\left(y_{i}, z_{i}\right) \leq 2 \varepsilon, \quad \text { for all } i \in \mathbf{N},
$$

no matter whether $\left\{y_{n_{k}}, z_{n_{k}}\right\} \subset X_{0}$ or not. Therefore, $f$ is equicontinuous. Theorem 5.1 is proven.

In the following we consider graph maps. A (finite) graph $G$ is a compact metric space that has a finite nonempty subset $V$ (the set of vertices) such that $G-V$ has only finitely many connected components all of which are homeomorphic to the open interval $(0,1)$. A continuous map from a graph $G$ to itself is called a graph map.

In $[8$, we proved the following theorem.

Theorem A. Let $G$ be a connected graph, and $f: G \rightarrow G$ be a continuous map. Then $f$ is pointwise recurrent if and only if $f$ is a periodic homeomorphism, or $G$ is a circle and $f$ is topologically conjugate to an irrational rotation of the unit circle $S^{1}$.

Noting that any periodic homeomorphism on a compact metric space and any irrational rotation of $S^{1}$ are equicontinuous, from Theorem A and Lemma 3.1 we obtain

Corollary 5.1. Every pointwise recurrent map of a connected graph is equicontinuous.

Theorem 5.2. Let $G$ be a connected graph with a metric d, and let $f: G \rightarrow G$ be a continuous map. Then the following four statements are equivalent:

(i) $f$ is equicontinuous;

(ii) $\bigcap_{n=1}^{\infty} f^{n}(G)=R(f)$;

(iii) $f \mid \bigcap_{n=1}^{\infty} f^{n}(G)$ is equicontinuous;

(iv) $f \mid \bigcap_{n=1}^{\infty} f^{n}(G)$ is a periodic homeomorphism, or is topologically conjugate to an irrational rotation of the unit circle $S^{1}$. 
Proof. (i) $\Rightarrow$ (ii) is clear, by Proposition 2.4.

(iii) $\Rightarrow$ (i). Since $G$ is compact and connected, for every $n \in \mathbf{N}, f^{n}(G)$ is also compact and connected. Write $G_{0}=\bigcap_{n=1}^{\infty} f^{n}(G)$. It follows from $G \supset f(G) \supset$ $f^{2}(G) \supset f^{3}(G) \supset \cdots$ that $G_{0}$ is a nonempty connected subgraph of $G$, and $f\left(G_{0}\right)=$ $G_{0}$. Put $V=G_{0} \cap \overline{G-G_{0}}$. Then $V$ is a finite set. Evidently, for every $k \in \mathbf{N}$, there exists $n_{k} \in \mathbf{N}$ such that $f^{n_{k}}(G) \subset G_{0} \cup B\left(V, 2^{-k}\right)$. Therefore, by Theorem 5.1, if $f \mid G_{0}$ is equicontinuous then $f$ is equicontinuous.

(ii) $\Rightarrow$ (iii). Let the connected subgraph $G_{0}=\bigcap_{n=1}^{\infty} f^{n}(G)$ be as above. If $G_{0}=R(f)$, then $f \mid G_{0}: G_{0} \rightarrow G_{0}$ is pointwise recurrent, and by Corollary 5.1, $f \mid G_{0}$ is equicontinuous.

(ii) $\Leftrightarrow($ iv) follows from Theorem A.

Remark 5.1. Let $\mathbf{C}$ be the complex plane, and let $D_{k}=\{z \in \mathbf{C}:|z| \leq k\}, k=1,2$. It is easy to construct a continuous map $f: D_{2} \rightarrow D_{2}$ such that $\bigcap_{n=1}^{\infty} f^{n}\left(D_{2}\right)=$ $R(f)=D_{1}$, and $f \mid D_{1}$ is a rotation, but $f$ itself is not equicontinuous. Therefore, in general, Theorem 5.2 is not true for spaces of dimension greater than 1 .

Remark 5.2. Let $G$ be a connected graph with a metric $d$, and let $G_{0}$ be a connected subgraph of $G$. Then any pointwise recurrent continuous map $f_{0}: G_{0} \rightarrow$ $G_{0}$ can be extended to an equicontinuous map $f: G \rightarrow G$. In fact, since $G_{0}$ is a retract of $G$, there exists a retraction $\gamma: G \rightarrow G_{0}$. Let $f=f_{0} \gamma: G \rightarrow G$. Then $f$ is a continuous extension of $f_{0}$. By Theorem 5.2, such an $f$ is equicontinuous.

If $G$ is a disconnected graph, then, for any continuous map $f: G \rightarrow G$, we can find a positive integer $k$ such that every connected component of $G$ intersecting $f^{k}(G)$ is an invariant set of $f^{k}$. Thus Theorem 5.2 can be easily generalized to the case that $G$ is not connected.

\section{ACKNOWLEDGEMENTS}

The author wishes to thank the referee for several valuable suggestions, especially a nice and short proof of Proposition 2.3 that has been adopted in this paper.

\section{REFERENCES}

[1] E. Akin, J. Auslander, and K. Berg, When is a transitive map chaotic?, Convergence in Ergodic Theory and Probability, Walter de Gruyter \& Co., Berlin, 1996, pp. 25-40. MR 97i:58106

[2] F. Blanchard, B. Host, and A. Maass, Topological complexity, Ergodic Theory Dynamical Systems, 20 (2000), 641-662. MR 2002b:37019

[3] L. S. Block and W. A. Coppel, Dynamics in one dimension, Lecture Notes in Mathematics, no. 1513, Springer-Verlag, New York, 1992. MR 93g:58091

[4] A. M. Blokh, The set of all iterates is nowhere dense in $C([0,1],[0,1])$, Trans. Amer. Math. Soc., 333 (1992), 787-798. MR 93c:26003

[5] W. Boyce, $\Gamma$-compact maps on an interval and fixed points, Trans. Amer. Math. Soc., 160 (1971), 87-102. MR 43:6374

[6] A. M. Bruckner and T. Hu, Equicontinuity of iterates of an interval map, Tamkang J. Math., 21 (1990), 287-294. MR 91m:58085

[7] J. Cano, Common fixed points for a class of commuting mappings on an interval, Proc. Amer. Math. Soc., 86 (1982), 336-338. MR 83h:54054

[8] Jie-Hua Mai, Pointwise recurrent graph maps, to appear. 
[9] Taixiang Sun , Equicontinuity of $\sigma$-maps, Pure and Applied Math. 16 (2000), No. 3, 9-14. (Chinese) MR 2001j:54049

[10] A. Valaristos, Equicontinuity of iterates of circle maps, Internat. J. Math. \& Math. Sci., 21 (1998), 453-458. MR 99c:54060

Institute of Mathematics, Shantou University, Shantou, Guangdong, 515063, PeoPle's Republic of ChinA

E-mail address: jhmai@stu.edu.cn 